\title{
MARKET SEgMENTATION: THE ROLE OF OPAQUE TRAVEL AgENCIES
}

\author{
DMITRY SHAPIRO \\ Belk College of Business \\ University of North Carolina-Charlotte \\ 9201 University City Boulevard \\ Charlotte, NC 28223-0001 \\ dashapir@uncc.edu \\ XIANWEN SHI \\ Department of Economics \\ University of Toronto \\ 150 St. George Street \\ Toronto, ON, Canada M5S 3G7 \\ xianwen.shi@utoronto.ca
}

This paper investigates the role of discount travel agencies such as Priceline and Hotwire in the market segmentation of the hotel and airline industries. These agencies conceal important characteristics of the offered services, such as hotel locations or flight schedules. We explicitly model this opaque feature and show that it enables service providers to price discriminate between those customers who are sensitive to service characteristics and those who are not. Service providers can profit from such discrimination despite the fact that the opaque feature virtually erases product differentiation and thus intensifies competition. The reason is that the intensified competition for less sensitive customers enables service providers to commit to a higher price for more sensitive customers, which leads to higher profits overall. This explains why airlines or hotels are willing to lose the advantage of product differentiation and offer services through discount travel agencies.

\section{INTRODUCTION}

In the past few years, the emergence of online electronic markets dramatically changed the leisure travel industry, making it one of the most developed online businesses. In the United States, online leisure

We are grateful to Mark Armstrong, Danielle Catambay, Stephen Morris, Artie Zillante, two anonymous referees and an anonymous coeditor whose valuable suggestions helped us to improve the paper.

(C) 2008, The Author(s)

Journal Compilation (C) 2008 Wiley Periodicals, Inc.

Journal of Economics \& Management Strategy, Volume 17, Number 4, Winter 2008, 803-837 
travel bookings more than tripled from 2001 to $2005,{ }^{1}$ and are expected to reach about $\$ 69$ billion in 2007, or 35\% of all online consumer spending. ${ }^{2}$ According to PhoCusWright, an independent consultancy, online leisure travel bookings in the United States will surpass offline bookings in volume for the first time in 2007. ${ }^{3}$

Expedia (who owns Expedia.com and Hotels.com), Travelocity and Orbitz are the three dominant online travel agencies (OTAs). Two niche players, Hotwire.com (acquired by Expedia in 2003) and Priceline.com, have emerged that offer services with $30-50 \%$ price discounts as compared to offline reservation prices or prices of other OTAs. These discount agencies have gained sizeable market shares. According to MarketMetrix, Priceline and Hotwire combined account for $6.7 \%$ of worldwide online hotel bookings in 2006, comparable to Expedia.com (10.4\%), Travelocity.com (6.8\%) and Orbitz.com (4.9\%). ${ }^{4,5}$ In November 2007 , Priceline reported a gross profit of $\$ 479$ million and gross travel bookings of $\$ 3.6$ billion worldwide for the first three quarters (a $58.9 \%$ and $40.6 \%$ increase compared to the same period in 2006, respectively). ${ }^{6}$

The defining feature of Priceline and Hotwire is that they do not tell customers certain itinerary details, such as brand, time of flight departure or exact hotel location, until the transaction is completed, and thus they are often referred to as "opaque" travel sites. ${ }^{7}$ Both sites used to offer opaque services exclusively, but recently, Priceline (in 2003) and Hotwire (in 2005) added the traditional transparent retail option with disclosed prices and itinerary details. Opaque sales nonetheless still remain a defining identity and major revenue source for both sites. In 2006, for example, Priceline reported that it "represented the substantial majority of our total revenues." 8

1. Source: The New York Times (Late Edition [East Coast]). May 30, 2005. p. C.6.

2. Source: http://www.comscore.com/press/release.asp?press=1545. Press release of comScore.com from July 30, 2007.

3. Source: http://store.phocuswright.com/phuontrovsee.html. PhoCusWright's U.S. Online Travel Overview, 2007.

4. Source: MarketMetrics.com, http://marketmetrix.com/en/default.aspx?s=research $\& \mathrm{p}=$ research 6 .

5. These figures likely underestimate the importance of Priceline and Hotwire in the leisure travel market because they include five-star hotels that are usually not offered by opaque sites (with the exception of Las Vegas). The figures also include sales in small cities where it is infeasible for opaque sites to operate-opaque sites need sufficiently many participating hotels to form an opaque product.

6. Priceline.com 2007 Q3 report, Form 10-Q, p. 23 and p. 26.

7. There is a slight difference between the two sites. Hotwire discloses the prices for opaque hotels or car rentals so consumers do not bid and simply decide whether to buy or not. Priceline, on the other hand, asks consumers to "Name Your Own Price" for the opaque service requested.

8. Priceline.com 2005 annual report Form 10-Q, p. 4, and 2006 annual report, Form 10-Q, p. 2. 
The opaque feature of Priceline and Hotwire has a strong impact on the competition between service providers. When the complete information about flights (or hotels) is available they are differentiated products. Hotels differ from each other in their identity, locations and amenities; flights differ in their departure times, the number of connections, and the length of layovers. It is well-known that product differentiation reduces competition and increases prices and profit (Hotelling, 1929). In contrast, products sold through Priceline and Hotwire are indistinguishable for customers and become essentially perfect substitutes, which leads to Bertrand competition and drives down both the price-cost margin and firms' profits.

The question is then: why would hotels and airline companies be willing to sell their products through Priceline or Hotwire and lose the advantage (and profit) that product differentiation gives them?

One explanation is that firms use OTAs with the opaque feature to respond to changes in demand without jeopardizing existing branding and pricing policies (formalized in Wang et al., 2006). Although this may be a part of the story we believe that this does not capture the whole picture. First, Priceline and Hotwire offer tickets during peak seasons (such as Christmas) and to popular destinations where the demand is traditionally high. Second, the amount of concealed information is more than the hotel or airline's identity, which also suggests that the purpose of these agencies is more than just a facility to anonymously respond to demand changes.

Another possible explanation is that opaque sites can help sellers reach new consumers with low valuations who otherwise remain outside the market. The low price of opaque services enables sellers to attract low value customers who are sensitive to price but less sensitive to service characteristics. On the other hand, the opaque feature prevents high value customers from switching to opaque channels because they would prefer to know itinerary details.

In this paper, we show that opaque agencies enable hotels and airlines to do much more than just attract low value consumers with cheap prices. In our model, they act as a "collusion device" to facilitate price discrimination between different types of customers and increase overall profits, even when the total market demand is perfectly inelastic.

We study the role of opaque travel agencies by using a variation of the Hotelling model. A model of horizontal differentiation is appropriate in this setting because both hotel and airline competitions are among service providers with similar qualities. First, Priceline and Hotwire disclose the star-ratings for hotels' service quality and amenities, so the competition is largely among hotels with similar qualities. Second, there is little quality difference among major airlines who provide 
the substantial majority of tickets to opaque sites..$^{9}$ In particular, all tickets sold through opaque agencies are economy (coach) class only. Furthermore, Priceline promises that flights have at most one stop each way, the layover is no longer than 3 hours, and no red-eye or off-peak flight are offered unless the traveler agrees to take one.

In this paper, we assume that there is a circle-shaped city where $N$ hotels are located (Salop, 1979). Consumers have two-dimensional types: location and transportation cost. Consumers' location type comes from the standard Hotelling model and is continuous. The transportation cost is binary-either high or low. We refer to consumers with a high transportation cost as business travelers, and a low transportation cost as leisure travelers.

We depart from the standard Hotelling's framework by explicitly modeling the opaque feature of Priceline and Hotwire. In our model, there is a single opaque travel agency that posts hotel prices and withholds hotel identities. Customers can make a reservation either via standard (nonopaque) travel agencies, or using the opaque travel agency. In the former case, customers can choose a specific hotel, and other things being equal, they would like to stay at the hotel that is closest to their preferred location. In the latter case, customers do not know the hotel's location and they simply prefer the hotel with the lowest price.

For a particular range of parameter values, we show that having an agency with the opaque feature enables hotels to separate high-type (business) travelers from low-type (leisure) travelers and to gain from this separation. The source of this gain comes from price discrimination. Without the opaque agency, hotels compete for both high and lowtype travelers through nonopaque reservation systems. The presence of the low-type in this market intensifies the competition and drives down the equilibrium price and profit. When an opaque travel agency (like Priceline or Hotwire) is introduced, a new equilibrium arises where high-type customers prefer to be served by agencies without the opaque feature and pay a high nonopaque price. Low-type customers, on the other hand, are served by the opaque agency that charges lower prices. The competition through the opaque agency is described by a Bertrand model, and so in the new equilibrium, hotel's competition for the low-type increases. However, competition in the more lucrative segment of the market-high-type travelers-decreases. It is still a Hotelling competition, but hotels no longer compete for the low-type. Only hightype customers buy differentiated products and in the equilibrium the

9. The five largest airline suppliers accounted for $82 \%$ of total tickets sold at Priceline in 2006. See Priceline.com 2006 annual report, p. 16. 
nonopaque price is higher. Consequently, as long as there is a sufficient number of high-type travelers, firms' overall profit increases.

Importantly, and perhaps somewhat surprisingly, it is the intensified competition for the low-type which enables hotels to decrease competition for the high-type. Leisure customers enjoy high surplus when being charged low opaque prices. To attract them to the nonopaque sector, a hotel would have to decrease the price too much to be profitable. As a result, in equilibrium hotels can sustain the high price of the nonopaque sector because they no longer use it to compete for leisure travelers.

The contribution of our paper is threefold. First, we formally model the opaque feature of Priceline.com and Hotwire.com and investigate its impact on the competition of the travel industry. Second, within our framework we find conditions on the degree of opacity that enables hotels and airlines to price discriminate using opaque OTAs. We show that the opacity level cannot be either too high or too low. If the opacity level is too high, leisure travelers would prefer to use standard reservation systems, while if the opacity level is too low, business travelers would find it optimal to purchase the opaque good. This finding is consistent with the fact that Priceline/Hotwire do not offer hotel rooms in small towns and divide large cities into zones. Third, our analysis helps explain why hotels and airlines are willing to use opaque channels to distribute their products. In particular, we identify the set of parameters for which the introduction of the opaque agency increases the overall profits of the industry.

\section{Literature REView}

In the literature there are only a few papers that focus on the effect of the opaque feature of Priceline and Hotwire. Most of them, like Wang et al. (2006), conduct analysis in the monopolistic setting, whereas we explicitly model the competition in the travel industry. To our knowledge, Fay (2008) is the only paper that models the opaque feature in the competitive environment. In his model, there are two firms and two types of consumers: those who are always loyal to a particular firm, and those whose preferences are distributed along the line between two firms as in the Hotelling model. The transportation cost of all consumers of the second type is the same.

Our paper is different from Fay (2008) in several aspects. First of all, we consider a more general framework with any number of firms. Here the number of firms can be interpreted as the degree of opacity of the products offered by discount agencies. Consequently, we are 
able to study the effect of opacity degree on the equilibrium prices with opaque OTAs. Second, we do not have loyal customers. Each consumer determines endogenously where to buy, and in particular, high-type customers might purchase the opaque product when it is cheap enough. Thus, in our framework we allow for a possibility that the opaque agency will cannibalize the profit of standard reservation systems. ${ }^{10}$ In contrast, cannibalization cannot happen in the model with loyal customers who only buy nonopaque products. Finally, the opaque agency in Fay (2008) always reduces firms' profit. Thus transfers from the opaque intermediary to firms are required in order for them to be willing to sell goods through the intermediary. In our setting, there are a range of parameter values when firms' profit strictly increases without any transfers.

Our approach is related to the Deneckere and McAfee (1996) paper on damaged goods. They show that producers can intentionally damage a portion of their good in order to price discriminate. Similarly, the opaque feature of Priceline and Hotwire is like damaging the original product by hiding important characteristics of a flight or a room. It then enables firms to discriminate between different types of consumers. Our model, however, is different from Deneckere and McAfee (1996) in two dimensions. First, we analyze a competitive market rather than a monopoly. Second, the damaging practice is done through a third party: an opaque travel agency.

This paper is also related to previous studies on facilitating practices. The role of the most-favored-customer (MFC) clause as a practice facilitating coordination in a price-setting duopoly has been studied extensively in the theoretical industrial organization literature (for example, Cooper, 1986; Holt and Scheffman, 1987), and the theory is confirmed by Scott Morton (1997a, 1997b), where she finds that the MFC clause adopted by Medicaid for reimbursement leads to an increase in drug prices. Another important facilitating device identified in the literature is trade restrictions. Krishna (1989) shows that quantitative restrictions, such as voluntary export restriction (VER), can impede competition, facilitate collusion and raise prices in a price-setting duopoly. Similarly, the introduction of opaque travel agency in our model can be regarded as a facilitating device that service providers can use to raise prices to business travelers in equilibrium. ${ }^{11}$

10. Cannibalization is a legitimate concern for many service provides. Northwest Airlines discontinued its relationship with Priceline on June 2002 for being increasingly concerned with Priceline's business model. Hotel industry expressed similar concern on the long-term risk of Priceline in cannibalization of sales from primary selling channels (see Wang et al., 2006).

11. One can also interpret the opaque service as a screening tool for the service providers to separate business travelers from leisure travelers. Thus our model is also 
Another paper that is related to ours is Granados et al. (2005). They examine the use of transparency strategy for Internet-based selling as a means for the firm to maximize the value of its selling activities. They descriptively argue how different online travel agencies adopt different level of transparency in product characteristics, price and supplier identity, and how consumers' willingness to pay varies with respect to different levels of transparency. Our model formalizes some of their ideas.

Generally, the novel practice of Hotwire.com and Priceline.com, has attracted much interest from economists and marketing researchers, and many of them study the "Name Your Own Price" feature of Priceline and its implication to seller's revenue and buyer's willingness to pay (for example, Hann and Terwiesch, 2003; Fay, 2004; Spann et al., 2004; Terwiesch et al., 2005). In contrast, we focus only on the opaque feature of Priceline and Hotwire and assume that opaque OTAs offer products at posted prices.

The rest of the paper is organized as follows. In Section 3, we formally describe a benchmark model without opaque travel agencies and solve for equilibria. In Section 4, we introduce an opaque agency and characterize all symmetric equilibria that arise in the new setting. We then compare the equilibrium profit among different equilibria and find which one is the most profitable. Section 5 concludes the paper. All the proofs are given in the Appendix.

\section{Model without Opaque agencies}

In this section we present a benchmark model that describes hotels' competition without opaque travel agencies. In the next section, we will introduce an opaque agency and compare the new equilibria with the benchmark.

Consider a circle-shaped city with $N$ hotels (Salop, 1979). The distance between any two adjacent hotels is $s$ and the length of the city is Ns. In what follows we assume that $s$ is fixed and does not depend on $N$. Consequently, as compared to standard models of horizontal differentiation, $N$ is not a measure of competition but rather a measure of the market size. We assume that hotels have unlimited room capacity, and the cost of providing one room is equal to $c$, which we normalize to 0 . Hotels compete with each other for travelers.

Travelers are described by their preferred location $x$ within the city and travel cost $t$. There are two groups of travelers: high-type (business)

related to the large screening literature. See Armstrong (2006) and Stole (2007) for recent surveys. 
travelers with travel cost $t_{H}$ and low-type (leisure) travelers with travel cost $t_{L}$. We assume that $t_{H} \geq t_{L}$. The type of each customer, thus, is a two-dimensional variable $(x, i)$, where $x \in[0, N s)$ and $i \in\{L, H\}$. Denote the set of all types as $T$. If a customer of type $(x, i) \in T$ stays at hotel $h$ and pays price $p$, his utility is

$u_{(x, i)}=v-t_{i} \cdot d(h, x)-p$,

where $d(h, x)$ is the distance between hotel $h$ and $x ; t_{i}$ is the cost of traveling a distance of one unit, and $v$ is the value of staying in a hotel. In what follows we will assume that the value of staying in the hotel $v$ is large enough so that all travelers will stay in some hotel. ${ }^{12}$

Location preferences of both high-type and low-type consumers are uniformly distributed along the circle. We assume that the total mass of consumers between two adjacent hotels is one. The fraction of business travelers among them is $\gamma$ and the fraction of leisure travelers is $1-\gamma$.

Notice that although we refer to $t_{i}, i \in\{L, H\}$, as travel cost, it can be interpreted more generally as the strength of preferences for a particular good characteristic. It could be the strength of consumers' preference for morning versus evening flights, or for one brand over another.

In what follows we shall restrict our attention to the symmetric equilibrium. In this equilibrium all hotels charge the same price $p_{n}$ where index $n$ stands for "no opaque agency". Proposition 1 describes this equilibrium and also specifies conditions under which this equilibrium exists.

\section{PROPOSITION 1:}

(i) In a symmetric equilibrium, if it exists, all hotels charge the price equal to

$$
p_{n}=\frac{s}{\gamma t_{L}+(1-\gamma) t_{H}} t_{L} t_{H}
$$

and all customers are served by the nearest hotel. The equilibrium profit $\pi_{n}=p_{n}$.

(ii) A symmetric equilibrium exists if and only if

$$
(1-\gamma) \frac{t_{H}}{t_{L}}+\gamma \leq \frac{\gamma / 2}{1-\gamma / 2-\sqrt{1-\gamma}} .
$$

The idea of the proof is quite straightforward. In the first part we use the standard Hotelling argument to show that the equilibrium price must be $p_{n}$ if a symmetric equilibrium exists. It is clear from

12. Given this assumption it is not essential whether $v_{L}=v_{H}$ or not. 
equation (1) that the higher is the share of leisure travelers, $1-\gamma$, the lower is the equilibrium price. Naturally, the presence of customers who are less sensitive to product differentiation intensifies the competition and drives down the equilibrium price and firms' profits.

In the second part we provide conditions under which the symmetric equilibrium exists. To see why it can fail to exist consider the case with $t_{H}>t_{L}=0$. It follows from part (i) of Proposition 1 that the equilibrium price and profits should be zero. Charging a small positive price then becomes a profitable deviation because a deviating hotel would serve a positive share of high-type customers. ${ }^{13}$ In fact, whenever $t_{H} / t_{L}$ is so high that (2) is violated it is always profitable for a particular hotel to increase its price and serve only high-type customers.

To get a further understanding of (2) note that for $\gamma$ close to 0 or 1 the upper bound on $t_{H} / t_{L}$ goes to infinity and so it becomes unprofitable to deviate unless $t_{H} / t_{L}$ is sufficiently large. Intuitively, when $\gamma$ is close to 0 , the number of business travelers is too small for hotels to profit from serving only high-type customers. Thus, unless $t_{H}$ is extremely large, hotels will not deviate from the symmetric equilibrium. When $\gamma$ is close to 1 then $p_{n}$ becomes sufficiently high so that, unless $t_{H}$ is sufficiently large, serving only high-type customers does not produce a higher profit. Putting it differently, when $\gamma$ is close to 1 leisure travelers are less of an impediment and the deviation becomes unprofitable.

\section{Model with an Opaque Agency}

In this section we will introduce an online travel agency with the opaque feature into the model. We will refer to travel agencies without the opaque feature as either transparent or standard agencies. The travel agency with the opaque feature will be referred to as the opaque or discount agency. Rooms reserved through the opaque (nonopaque) agency will be often called opaque (nonopaque) rooms.

Travelers can either use a standard reservation system or the opaque agency. In the former case travelers can choose a hotel where they prefer to stay. In the latter case, hotels' identity is revealed only

13. The fact the symmetric equilibrium in pure strategies fails to exist for large travel cost ratios is not unique to our model and holds in other similar models. In Ellison (2005), for example, there are two types of customers with different marginal utilities of income. That is, a person paying price $p$ for a good receives disutility of $\alpha_{i} p$ where $i \in\{l, h\}$ and $\alpha_{l}>\alpha_{h}$. In Ellison's model the symmetric equilibrium exists only for a particular range of $\alpha_{l} / \alpha_{h}$ that is bounded from the above by 10.66 (Ellison, 2005, p. 632). If the $\alpha$-ratio is higher than 10.66 there exists a profitable deviation where a firm raises its price and serves only high-type customers. 
after the transaction is completed and it could be any one of participating hotels. Thus the set of travelers' choices is $C_{t}=$ \{opaque agency, $1, \ldots, N\}$. In what follows we will focus on equilibria where all hotels participate. Consequently, a higher $N$ implies that customers have less precise information about the location of the opaque room. This suggests an alternative interpretation of $N$ as a measure of the opacity level for rooms sold through the opaque agency. A higher $N$ implies that more information is concealed and so the opacity level is higher.

The timing of the game is as follows. First, travelers observe their types. Then hotels publicly announce list prices $\left\{p_{h}\right\}_{h=1}^{N}$ and decide whether they are willing to participate in the opaque sales or not. Those hotels that decide to participate privately submit discounted prices $\left\{q_{h}\right\}_{h=1}^{N}$ to the opaque agency. The opaque agency posts discounted prices but conceals hotel identities. Travelers learn which hotels participate in opaque sales ${ }^{14}$ and observe $\left\{p_{h}, q_{h}\right\}$. Afterwards they decide whether to reserve an opaque or nonopaque room and for the latter case they also choose the hotel to stay in.

As the timing of the game suggests, a hotel's strategy is a pair $\left(p_{h}, d_{h}\right)$, where $p_{h}$ is a publicly available rate and $d_{h}$ is either a discounted price $q_{h}$ or a nonparticipating decision. A traveler's strategy is a mapping $\sigma_{(x, i)}$ such that for a given vector of hotel's strategies $\left\{\left(p_{h}, d_{h}\right)\right\}_{h=1}^{N}$ it determines a reservation choice in $C_{t}$. The mapping is indexed by traveler's type $(x, i)$. Finally, the opaque agency in our model is not strategic and its role is reduced to posting hotel prices while concealing hotel identities. In particular, we do not model the competition between opaque agencies and their price-setting strategies. This is done for two reasons. First, Priceline and Hotwire adopt very different pricing strategies and their details are not well known. Therefore, it would be too subtle and complicated to precisely model the competition between the two opaque sites. Second, the focus of the paper is the role of opaque agencies in market segmentation and introducing a strategic component in their behavior would only increase the complexity of the paper without adding much insight.

14. That is, we assume that a hotel's participation decision is publicly observable to travelers. Therefore, if a particular hotel withdraws from the opaque agency, it will affect travelers' perception of the opacity level of the opaque service. For example, if hotel $h$ does not participate in the opaque agency, then for travelers located at point $h$ the opaque agency becomes less attractive. Alternatively, we can assume that hotel's participation is private so that the withdrawal of one hotel has no effect on consumers' perceived opacity level. For instance, in case of flight tickets the main source of opacity is concealed itinerary information and is largely unaffected by a withdrawal of one airline. Under this alternative assumption, the results are qualitatively the same. However, the set of parameters for which different types of equilibria exist is larger. 
DEFINITION 1. A strategy profile $\left(\left\{p_{h}, d_{h}\right\}_{h=1}^{N},\left\{\sigma_{(x, i)}\right\}_{(x, i) \in T}\right)$ is an equilibrium in the model with an opaque travel agency if:

(a) Given the behavior of other hotels, $\left(p_{-h}, d_{-h}\right)$, and customers' strategies $\left\{\sigma_{(x, i)}\right\}_{(x, i) \in T}$, hotel $h$ chooses list price $p_{h}$ and $d_{h}$ to maximize its profit;

(b) Given hotel prices $\left\{p_{h}, q_{h}\right\}_{h=1}^{N}$ a traveler of type $(x, i)$ chooses a strategy $\sigma_{(x, i)}$ to maximize his utility. Specifically, for each price vector a traveler has to decide whether to get a room in a particular hotel or whether to reserve a room through the opaque agency.

In the paper, we will restrict our attention only to symmetric equilibria in which all hotels participate in opaque sales. In these equilibria $p_{1}=\cdots=p_{N}$ and $q_{1}=\cdots=q_{N}$.

From the buyers' point of view, rooms offered by different hotels through the opaque agency are perfect substitutes. Thus when selling rooms via the opaque agency, hotels lose the advantage of product differentiation and the competition drives the discounted price down to the marginal cost. To see this, notice that if a customer of type $(x, i)$ prefers the opaque room his total cost will be $q_{h}+t_{i} \cdot \mathbb{E}[d]$, where $\mathbb{E}[d]$ is the expected distance that the customer will have to travel. Because $\mathbb{E}[d]$ is the same for all hotels, the customer will always choose the one with the cheapest price. It follows from the logic of Bertrand competition that the discounted price must be equal to the marginal cost.

The fact that hotels compete for customers not only through standard reservation systems but also through the discount agency is somewhat extreme because it leads to zero profits from the opaque sales. Nonetheless, as Proposition 6 shows, having the discount agency can increase overall profit even when the profit from opaque sales is zero. Thus, if we change the way hotels compete at the opaque level so that they do earn positive profits from opaque transactions, then the introduction of the opaque agency would become even more profitable.

We will assume that when hotels charge the same discounted price they equally split all customers. From the customer's point of view, this means that the probability of staying at a particular hotel is the same and is equal to $1 / N$. Assume that $N$ is even and take a customer located at distance $0 \leq x \leq s / 2$ from the nearest hotel. His expected travel cost is

$$
\begin{aligned}
& \frac{1}{N}\left[x+(s-x)+(x+s)+(2 s-x)+\cdots+\left(x+\left(\frac{N}{2}-1\right) s\right)\right. \\
& \left.\quad+\frac{N}{2} s-x\right]=\frac{N}{4} s .
\end{aligned}
$$


Not surprisingly, larger $N$ leads to a larger expected cost. In other words, a higher opacity degree of rooms leads to a higher disutility from using the opaque agency. Another important observation is that for even $N$ the expected travel cost does not depend on $x$. This fact will dramatically simplify our analysis and in what follows we will keep the assumption of even $N$.

\subsection{CLASSIFICATION OF EQUiLIBRIA}

In the model with an opaque agency, customers are divided between opaque and nonopaque market segments. Technically, there are many ways they can be split between the two. However, as we show below most of them are not possible in equilibrium.

- All customers reserve opaque rooms. This is not an equilibrium. Indeed, in this case all hotels earn zero profit. Hotel $h$ could profitably deviate by charging a small positive list price which would attract high-type customers located at or close to $h$.

- Nobody reserves opaque rooms. For some parameter values this could be an equilibrium. Obviously, this equilibrium would coincide with the one found in Section 3 and, in particular, hotels earn the same profit in both equilibria. Given this and the fact that Priceline and Hotwire complete millions of transactions annually we will not consider this case in the paper.

- Some high-type travelers reserve opaque rooms. The next proposition shows that this is impossible as long as $N$ is not too small.

Proposition 2: If $N \geq 4$ then in any symmetric equilibrium all customers of the high type prefer to use the nonopaque agency.

The intuition behind this result is straightforward. When $N$ is large the uncertainty for high-type customers about the opaque product becomes high. Consequently, hotels find it easier and more profitable to attract business customers to the standard reservation system.

Because we are only interested in the equilibria where some travelers are served by the opaque agency there are two possibilities left.

- Full separation. All high-type customers reserve through standard travel agencies, and all low-type customers use the opaque service.

- Partial separation. All high-type customers and some (but not all) low-type customers reserve through standard travel agencies. The remaining low-type customers use the opaque service.

Below we will solve for equilibria with full and partial separations. We analyze the former in Section 4.2 and the latter is studied in 
Section 4.3. It was already established that the equilibrium discounted price is equal to the marginal cost which is zero. Thus what is left to do is to find the list price and verify that it determines an equilibrium.

\subsection{EQUILIBRIUM WITH FULL SEPARATION}

DEFINITION 2. The list price $p_{f}$ is an equilibrium price in an equilibrium with full separation if the following conditions are satisfied:

(a) Business travelers who are located exactly in the middle between two hotels prefer to use standard reservation services:

$$
p_{f}+\frac{1}{2} s t_{H} \leq \frac{1}{4} N s t_{H} .
$$

(b) Leisure travelers whose location preferences coincide with one of the hotels prefer to use the opaque agency:

$$
\frac{1}{4} N s t_{L} \leq p_{f} \text {. }
$$

(c) For each hotel $h$, list price $p_{f}$ maximizes its profit when all other hotels charge $p_{f}$;

(d) Each hotel finds it profitable to participate in opaque sales.

It follows from (a) that all business travelers will use standard agencies, from (b) it follows that all leisure travelers will use the opaque agency. Conditions (c) and (d) guarantee that hotels do not have an incentive to deviate from the equilibrium.

\section{PROPOSITION 3:}

(i) If there exists an equilibrium with full separation then the equilibrium list price is $p_{f}=s t_{H}$;

(ii) The full separation equilibrium with $p_{f}=s t_{H}$ exists if and only if the following conditions are satisfied: $N \geq 6$, and

$$
\frac{t_{H}}{t_{L}} \geq \frac{N}{N-1}\left(\frac{N}{4}+\frac{1-\gamma}{2 \gamma}\left(\frac{N}{4}\right)^{2}\right) .
$$

Part (i) explicitly assumes that only high-type customers use the nonopaque agency, in which case an immediate extension of the standard Hotelling logic shows that $p_{f}=s t_{H}$. In order for $p_{f}$ to be an equilibrium price, $p_{f}$ should satisfy requirements (a)-(d) in Definition 2 which leads to conditions specified in part (ii). Intuitively, if we treat $N$ as the measure of the opacity degree part (ii) states that the equilibrium with full separation exists only for moderate opacity levels. If opacity is too low, that is, $N<6$, business travelers will switch to the opaque 
agency because the uncertainty about hotel locations is not as large as the difference between the list and discounted price. If the opacity level is so high such that condition (3) is violated, then both leisure travelers and hotels will deviate from the full separation. Leisure travelers will find the location uncertainty to be too big and switch to the nonopaque service. Hotels will find it profitable to cut the price and serve some low-type customers directly through nonopaque channels. Other requirements that condition (3) imposes on parameters is that, $t_{H} / t_{L}$ and $\gamma$ should be sufficiently high. The former means that business travelers should be sufficiently different from leisure travelers and the latter means that there should be sufficiently many of them.

Interestingly, at least to some extent, it is the intense Bertrand competition on the opaque level that enables hotels to charge higher prices through their standard reservation systems. Very low opaque prices make it unprofitable for hotels to deviate and attract low-type customers. The high nonopaque price can then be sustained in the equilibrium. To see this, assume that the discount price is $q$ and consider a leisure traveler whose location preference coincides with the location of hotel $h$. The total cost of reserving an opaque room is $q+N / 4 s t_{L}$. The total cost of reserving a nonopaque room at hotel $h$ is $p_{h}$ (the list price of hotel $h$ plus zero travel cost). When $p_{h}<q+N / 4 s t_{L}$ this traveler and other leisure travelers close to him will prefer to reserve rooms in hotel $h$. Thus, the higher the opaque price is, the easier it becomes for hotels to attract low-type customers to their nonopaque channels.

Clearly, it would be incorrect to claim that the Bertrand competition on the opaque level is necessary to prevent hotels from deviation. In particular, depending on parameters the full separation equilibrium will still exist if the opaque agency charges a small fee for each transaction. The main message is rather that in order for hotels to charge a high list price in equilibrium, leisure travelers should be charged low prices. Bertrand competition is just an extreme yet easy-to-implement way of doing that.

\subsection{EQUILIBRIUM WITH PARTIAL SEPARATION}

In this section we will describe equilibria when some (but not all) leisure travelers reserve opaque rooms.

DEFINITION 3. The list price $p_{p s}$ is an equilibrium price in a symmetric equilibrium with partial separation if the following conditions are satisfied:

(a) Business travelers who are located exactly in the middle between two hotels prefer to use standard reservation services:

$$
p_{p s}+\frac{1}{2} s t_{H} \leq \frac{1}{4} N s t_{H} .
$$




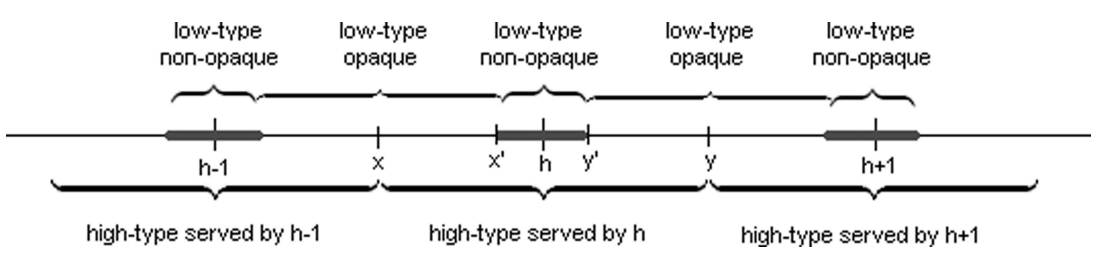

FIGURE 1. TRAVELER $x$ IS A HIGH-TYPE TRAVELER INDIFFERENT BETWEEN STAYING AT $h$ AND $h-1$. TRAVELER y IS A HIGH-TYPE INDIFFERENT BETWEEN $h$ AND $h+1$. TRAVELERS $x^{\prime}$ AND $y^{\prime}$ ARE LEISURE TRAVELERS INDIFFERENT BETWEEN A ROOM AT $h$ AND AN OPAQUE ROOM. THE DISTANCE FROM TRAVELERS $x^{\prime}$ AND $y^{\prime}$ TO HOTEL $h$ IS d'

(b) Leisure travelers located at distance $d^{\prime} \in(0, s / 2)$ from the nearest hotel are indifferent between reserving opaque and nonopaque rooms.

$$
\frac{1}{4} N s t_{L}=p_{p s}+d^{\prime} t_{L}
$$

(c) For each hotel $h$, list price $p_{p s}$ maximizes its profit when all other hotels charge $p_{p s}$.

(d) Each hotel finds it profitable to participate in opaque sales.

Figure 1 shows choices that will be made by customers in an equilibrium with partial separation. Hotel $h$ serves all low-type customers whose distance to hotel $h$ is less than $d^{\prime}$ and only them. From $\left(I C_{L}^{\prime}\right)$ we know that

$d^{\prime}=\frac{\frac{1}{4} N s t_{L}-p_{h}}{t_{L}}$.

The set of business travelers served by hotel $h$ is determined by locations of two customers: $x$ who is indifferent between $h$ and $h-1$ and $y$ who is indifferent between $h$ and $h+1$. From indifference conditions their locations are:

$x=\frac{p_{h-1}-p_{h}}{2 t_{H}}+\frac{1}{2} s ; \quad y=\frac{p_{h+1}-p_{h}}{2 t_{H}}+\frac{1}{2} s$.

Given that hotels $h-1$ and $h+1$ set price equal to $p_{p s}$ the maximization problem for hotel $h$ becomes

$$
\max _{p_{h}}\left\{\frac{\gamma}{s}\left(\frac{p_{p s}-p_{h}}{t_{H}}+s\right) p_{h}+2 \frac{1-\gamma}{s} \frac{\frac{1}{4} N s t_{L}-p_{h}}{t_{L}} p_{h}\right\} .
$$


The first-order condition and the symmetry restriction imply that $p_{p s}=\frac{\gamma+\frac{1}{2}(1-\gamma) N}{\gamma t_{L}+4(1-\gamma) t_{H}} s t_{L} t_{H}$

For $p_{p s}$ to be an equilibrium it has to satisfy $\left(I C_{H}^{\prime}\right)$ and $\left(I C_{L}^{\prime}\right)$. In particular, it should be the case that $d^{\prime}$ as determined by (4) should belong to $(0, s / 2)$. If $d^{\prime} \leq 0,\left(d^{\prime} \geq s / 2\right)$ then all leisure consumers would use the opaque (nonopaque) agency.

Proposition 4: If $N \geq 6$, then $p_{p s}$ satisfies $\left(I C_{H}^{\prime}\right)$.

Intuitively, when the number of hotels is sufficiently large (as large as 6), the expected travel cost for the business travelers will be high, which would make opaque reservations less attractive for them.

Proposition 5: $\left(I C_{L}^{\prime}\right)$ is satisfied if and only if either one of the following conditions holds

$$
\frac{N-4}{N-2}<\gamma \leq \frac{N}{N+2} \text { and } \quad \frac{1}{2} \frac{(N-2) \gamma}{\gamma(N-2)-(N-4)}<\frac{t_{H}}{t_{L}}
$$

$\frac{N}{N+2}<\gamma<1$ and $\frac{1}{2} \frac{(N-2) \gamma}{\gamma(N-2)-(N-4)}<\frac{t_{H}}{t_{L}}<\frac{1}{4} \frac{N \gamma}{\gamma-\frac{1}{2}(1-\gamma) N}$.

The intuition behind Proposition 5 is as follows. When $\gamma$ is small (less than $(N-4) /(N-2)$ ) hotels will always find it optimal to attract all leisure travelers to nonopaque agencies. When $\gamma$ is moderately high then, as long as there is a substantial difference in travel costs, the partial equilibrium will exist. However, when $\gamma$ is very high (larger than $N /(N$ $+2)$ ) the difference in travel costs cannot be too large. Otherwise hotels will not be interested in serving the low-type at all (at least not through standard reservation systems).

Propositions 4 and 5 characterize necessary conditions for $p_{p s}$ to be a partial separation equilibrium. If, given $p_{p s}$, all hotels prefer to participate in the opaque sales, then $p_{p s}$ will indeed be an equilibrium. In the next section we will show that whenever these necessary conditions are satisfied the partial separation equilibrium is always less profitable than the equilibrium without the opaque agency. Therefore, we do not derive a full set of conditions for the existence of the partial separation equilibrium.

\subsection{PROFIT COMPARISON}

In previous sections we have solved for equilibria with full and partial separation and described the necessary conditions under which they 
exist. In this section we are interested in how hotel profits in these equilibria compare to profits in the equilibrium without the opaque agency.

It is worth mentioning that in all three equilibria, the size of the market is the same. Given our assumptions, the total demand for travel services is perfectly inelastic and is always equal to a total mass of travelers, $N$. However, in different equilibria different groups of customers pay different prices. In the equilibrium without the opaque agency all customers pay the same price $p_{n}$ (see (1)). In the equilibrium with full separation, leisure travelers pay price equal to the marginal $\operatorname{cost} 0$, and business travelers pay higher price, $p_{f}=s t_{H}$. Finally, in the equilibrium with partial separation, some leisure travelers pay price zero, whereas all other customers pay higher price $p_{p s}$ (see (6)).

The next proposition contains the main result of this paper as it compares the profits across different types of equilibria. In particular, it characterizes conditions under which the equilibrium with full separation is more profitable than the equilibrium without an opaque agency.

\section{PROPOSITION 6:}

(i) Assume that parameters are such that both the equilibrium without an opaque agency and the equilibrium with the full separation exist. Then the latter is more profitable if and only if

$$
\frac{t_{H}}{t_{L}}>\frac{1+\gamma}{\gamma} \text {. }
$$

(ii) For any $N \geq 6$ the set of parameters for which both equilibria exist AND the equilibrium with full separation is more profitable is not-empty.

(iii) The equilibrium with partial separation, if it exists, is less profitable than the equilibrium without an opaque agency.

The first part of the proposition determines the conditions when the equilibrium with full separation is more profitable than the equilibrium without the opaque agency. The condition itself immediately follows from a comparison of the profits in two equilibria and is very intuitive. The full separation profit $\pi_{f}=\gamma s t_{H}$ is determined by the amount of customers served, $\gamma$, and the price they pay $s t_{H}$. Consequently, for $\pi_{f}$ to be higher it should be the case that $\gamma$ and $t_{H}$ (as compared to $t_{L}$ ) are sufficiently high. Another interpretation of (7) is that for each particular level of $\gamma$ the travel cost ratio should be high enough. The lower (higher) is $\gamma$, the higher (lower) should be the difference in travel costs in order for the full separation to be more profitable.

The second part of the proposition verifies that the conditions for the existence of both equilibria and the conditions under which the full 


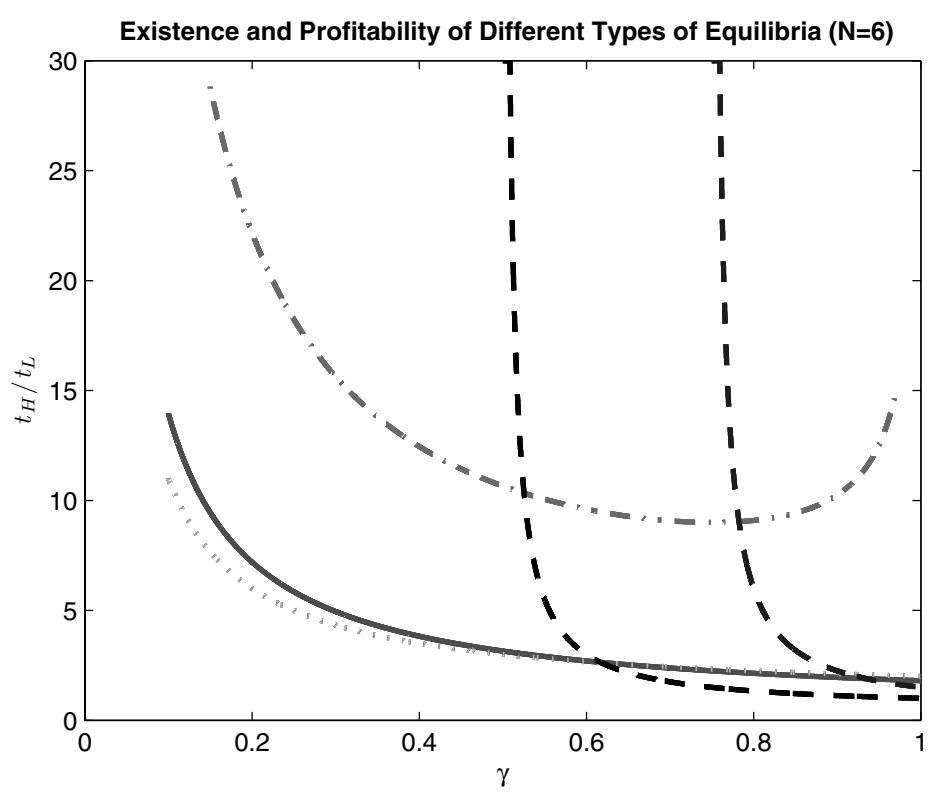

FIGURE 2. THE DASH-DOTTED LINE ON THE TOP REPRESENTS CONDITION (2). FOR TRAVEL-COST RATIOS BELOW THIS LINE THE SYMMETRIC EQUILIBRIUM EXISTS. THE SOLID LINE ON THE BOTTOM REPRESENTS CONDITION (3). FOR TRAVEL COST RATIOS ABOVE THE SOLID LINE THE SYMMETRIC EQUILIBRIUM EXISTS. THE DOTTED LINE ON THE BOTTOM REPRESENTS CONDITION (7). FOR TRAVEL COST RATIOS ABOVE THIS LINE THE EQUILIBRIUM WITH FULL SEPARATION IS MORE PROFITABLE. FINALLY, THE TWO NEARLY VERTICAL DASHED LINES BOUND THE AREA WHERE THE EQUILIBRIUM WITH PARTIAL SEPARATION EXISTS

separation equilibrium is more profitable are not mutually exclusive. There are four conditions involved: condition (2) from Proposition 1 guarantees that the equilibrium with full separation exists. Conditions $N \geq 6$ and (3) of Proposition 3 guarantee that the equilibrium with full separation exists and finally condition (7) of the current proposition guarantees that the full separation is more profitable. Figure 2 helps visualize these conditions for $N=6$. Both equilibria exist and the full separation is more profitable in the area below the dash-dotted line and above the upper envelope of the solid and the dotted lines.

The last part of Proposition 6 shows that the equilibrium with partial separation, if it exists, is always less profitable than the equilibrium without the opaque agency. 
The most important result in Proposition 6 is part (i) that determines when introducing an opaque agency leads to a new equilibrium with higher profit. The source of the profit increase does not come from new customers who are attracted to the market by cheap prices. As we mentioned earlier, in our model the size of the market does not respond to prices. The source of the extra profit comes from hotels' ability to separate different types of customers and to make the hightype pay higher prices. Importantly, it is the opaque agency that enables hotels to do that. Without the opaque agency hotels would compete for the low-type thereby driving the equilibrium price down. With the opaque agency, the low-type pays an extremely low price and it is not profitable for hotels to compete for them via nonopaque channels. This enables hotels to sustain a higher list price paid by business travelers and to increase the overall profit.

Clearly, hotels will be interested in the opaque agency only if the profit in the new equilibrium is higher than in the equilibrium without the opaque agency. This will occur when the equilibrium with full separation exists and is more profitable. In particular, there should be sufficiently many business customers on the market and travel costs of business and leisure customers should be sufficiently different. A possible caveat is that if parameters are such that the equilibria with both full and partial separation exist then there is a multiplicity of equilibria with the former being more profitable than the benchmark, and the latter being less profitable. However, if hotels can coordinate on a particular equilibrium then they will coordinate on the equilibrium with full separation as this is the most profitable one. On Figure 2 the set of parameters for which all three equilibria might co-exist is bounded by the solid line on the bottom, the dash-dotted line on the top and two dashed lines on the sides.

Finally, from our analysis we can elucidate the role of the degree of opacity. Except for the profit-inferior equilibrium with partial separation $N$ has no effect on the equilibrium prices and profits. $N$ does affect, however, the set of parameters for which the equilibria with partial and, most importantly, full separation exist. As established in Proposition 3, for the equilibrium with full separation to exist, the degree of opacity cannot be too low or too high. A low degree of opacity would encourage business travelers to switch to the opaque agency, in which case, the opaque OTA would cannibalize on standard distribution channels and lead to a decline in profit. A high degree of opacity would make it unprofitable for leisure customers to use the opaque agency. In this case, the equilibrium with full separation will not exist, and the introduction of the opaque agency will fail to increase hotels' profits. 


\section{CONCLUSION}

The main goal of our paper is to provide a rationale of why firms that produce differentiated goods decide to deliver goods through opaque services such as Priceline or Hotwire, and to investigate how these services affect competition. Due to the opaque feature of Priceline and Hotwire, rooms from different hotels and flights of different airlines are indistinguishable, so the competition is head to head. It may seem counterintuitive for firms to join opaque services because it intensifies competition. In the literature potential explanations to this puzzle include a possibility that hotels can use Priceline and Hotwire to adjust for seasonal changes in demand or to attract new customers with very high price sensitivity.

In this paper, we take a different approach. We assume that the market size is fixed so that firms cannot attract more customers by lowering prices. Nonetheless, as we show, firms may still prefer an equilibrium with the opaque agency over the equilibrium without it. This occurs because the opaque agency increases competition for low-type customers who are less sensitive to particular characteristics of the good (e.g., a hotel location). On the other hand, the existence of an opaque agency decreases competition for the more lucrative segment of the market-high-type customers who are sensitive to differences in concealed characteristics. As we show, under some natural conditions, increased profits from high-type customers outweigh losses from low-type customers and so the total profits increase.

\section{APPENDIX: ProOfS}

Proof of Proposition 1

(i) In the symmetric equilibrium, if it exists, hotel $h$ serves both types of customers and directly competes only with the adjacent hotels $h-1$ and $h+1$.

Denote the price charged by hotel $h$ as $p_{h}$. Business and leisure customers indifferent between hotels $h+1$ and $h$ are located at $x_{H}$ and $x_{L}$ from hotel $h$, where $x_{H}$ and $x_{L}$ are given by

$x_{H}=\frac{p_{h+1}-p_{h}}{2 t_{H}}+\frac{1}{2} s \quad$ and $\quad x_{L}=\frac{p_{h+1}-p_{h}}{2 t_{L}}+\frac{1}{2} s$.

Similarly, business and leisure customers indifferent between hotels $h$ and $h-1$ are located at $y_{H}$ and $y_{L}$ from hotel $h$, where $y_{H}$ and $y_{L}$ are 
given by

$y_{H}=\frac{p_{h-1}-p_{h}}{2 t_{H}}+\frac{1}{2} s \quad$ and $\quad y_{L}=\frac{p_{h-1}-p_{h}}{2 t_{L}}+\frac{1}{2} s$.

Therefore, hotel $h$ 's profit is

$$
\begin{aligned}
\pi_{h}\left(p_{h}, p_{h+1}, p_{h-1}\right)= & \frac{\gamma}{s}\left(x_{H}+y_{H}\right) p_{h}+\frac{1-\gamma}{s}\left(x_{L}+y_{L}\right) p_{h} \\
= & \frac{\gamma}{s}\left(\frac{p_{h-1}+p_{h+1}-2 p_{h}}{2 t_{H}}+s\right) p_{h} \\
& +\frac{1-\gamma}{s}\left(\frac{p_{h-1}+p_{h+1}-2 p_{h}}{2 t_{L}}+s\right) p_{h} .
\end{aligned}
$$

The necessary first-order conditions for the equilibrium prices are:

$$
p_{h}=\frac{p_{h-1}+p_{h+1}}{4}+\frac{1}{2} \frac{s t_{L} t_{H}}{\gamma t_{L}+(1-\gamma) t_{H}}, h=1, \ldots, N
$$

It can be immediately verified that prices

$$
p_{1}=\cdots=p_{N}=\frac{s}{\gamma t_{L}+(1-\gamma) t_{H}} t_{L} t_{H}
$$

satisfy the first-order conditions and are the only candidates for the symmetric equilibrium prices.

(ii) The rest of the proof is devoted to finding conditions under which $p_{n}$ constitutes an equilibrium. Recall that in part (i) we explicitly assumed that hotel $h$ serves both types of customers and competes only with the adjacent hotels. Thus there are two possible deviations that have not been accounted for in part (i). First, if hotel $h$ decreases its price so much that it competes with hotels that are not adjacent. For this to happen $p_{h}$ should be less than $p_{n}-s t_{L}$. Second, if hotel $h$ raises the price so much that it serves only business customers. This will occur when $p_{h}>p_{n}+s t_{L}$.

We start with the first deviation and show that it is never profitable. Assume that all other hotels charge prices $p_{n}$ and hotel $h$ charges $p_{h}<$ $p_{n}-s t_{L}$. Business customer located at point $h-1$ prefers hotel $h-1$ to $h$ if and only if $p_{n} \leq p_{h}+s t_{H}$. If $p_{h}=0$ the inequality above becomes

$$
\frac{s}{\gamma t_{L}+(1-\gamma) t_{H}} t_{L} t_{H} \leq s t_{h}
$$

which is always satisfied. Thus even if hotel $h$ decreases its price to zero it will compete for business customers only with the adjacent hotels. 
Consequently, hotel profit function has the following form:

$$
\pi_{h}\left(p_{h}\right)=\left\{\begin{array}{l}
\frac{\gamma}{s}\left(\frac{2 p_{n}-2 p_{h}}{2 t_{H}}+s\right) p_{h}+\frac{1-\gamma}{s}\left(\frac{2 p_{n}-2 p_{h}}{2 t_{L}}+(k+1) s\right) p_{h} \\
\quad \text { if } p_{n}-k s t_{L} \geq p_{h} \geq p_{n}-(k+1) s t_{L} \\
\frac{\gamma}{s}\left(\frac{2 p_{n}-2 p_{h}}{2 t_{H}}+s\right) p_{h}+(1-\gamma) N p_{h} \\
\quad \text { if } p_{n}-(N / 2) s t_{L} \geq p_{h}
\end{array}\right.
$$

The first line shows $h$ 's profit when it competes with a hotel at distance $(k+1) s$ from $h$ 's location. This is for each $k$ between 1 and $(N / 2)$ -1 . The second line is $h^{\prime}$ s profit when it serves all leisure customers. We will show that the profit function is strictly increasing on the range $p_{h} \leq p_{n}-s t_{L}$ which will imply that $p_{h}<p_{n}-s t_{L}$ cannot be a profitable deviation.

To see that the profit function is increasing notice that it consists of $N / 2$ concave parabolas. Parabolas described by the first line achieve their maximum at

$$
\hat{p}_{h}=\frac{p_{n}+p_{n}(\gamma+(k+1)(1-\gamma))}{2}>p_{n} .
$$

The parabola described by the second line achieves its maximum at

$$
\bar{p}_{h}=\frac{\gamma p_{n}+(\gamma+(1-\gamma) N) s t_{H}}{2 \gamma}>p_{n} .
$$

Thus each parabola is an increasing function on the range where it determines the profit and so $\pi_{h}$ is an increasing function when $p_{h}<p_{n}-s t_{L}$. This proves that it is never profitable for a hotel to decrease the price and try to compete with hotels that are not adjacent.

The second possible deviation for hotel $h$ is to dramatically increase $p_{h}$ so that it serves only high-type customers. This happens whenever $p_{h}>p_{n}+s t_{L}$ and hotel's profit on this interval is

$\pi_{h}\left(p_{h}\right)=\frac{\gamma}{s}\left(\frac{2 p_{n}-2 p_{h}+2 s t_{H}}{2 t_{H}}\right) p_{h}$.

The profit function is strictly concave in $p_{h}$ and is maximized at $p_{h}^{\prime}=$ $\left(p_{n}+s t_{H}\right) / 2>p_{n}$. Thus hotel $h$ will have a profitable deviation if and only if $p_{h}^{\prime}>p_{n}+s t_{L}$ and $\pi_{h}\left(p_{h}^{\prime}\right)>\pi_{h}\left(p_{n}\right)$. The first condition says that a leisure customer located at point $h$ prefers to be served by hotel $h-1$ and thus hotel $h$ serves only business travelers when charging $p_{h}^{\prime}$. The second condition states that charging $p_{h}^{\prime}$ is profitable. If either one of two 
conditions fails, $p_{n}$ constitutes a symmetric equilibrium and hotels do not have profitable deviations.

Condition $p_{h}^{\prime}>p_{n}+s t_{L}$ is equivalent to

$$
\frac{t_{H}}{t_{L}}>\frac{3}{2}+\frac{1}{2} \sqrt{\frac{9-\gamma}{1-\gamma}} \text {. }
$$

Condition $\pi\left(p_{h^{\prime}}\right)=\frac{\gamma}{4 s t_{H}}\left(p_{n}+s t_{H}\right)^{2}>p_{n}=\pi_{h}\left(p_{n}\right)$ is equivalent to

$$
(1-\gamma) t_{H} / t_{L}+\gamma>\frac{\gamma / 2}{1-\gamma / 2-\sqrt{1-\gamma}} \text {. }
$$

Define

$$
l_{1}=\frac{3}{2}+\frac{1}{2} \sqrt{\frac{9-\gamma}{1-\gamma}} \text { and } l_{2}=\frac{1}{1-\gamma}\left(\frac{\gamma / 2}{1-\gamma / 2-\sqrt{1-\gamma}}-\gamma\right)
$$

so that we can rewrite conditions (A2) and (A3) as $t_{H} / t_{L}>l_{1}$ and $t_{H} / t_{L}>$ $l_{2}$, respectively. With long and tedious algebra that we omit one can show that $l_{1}<l_{2}$ for any $0<\gamma<1$.

Now it is easy to see that hotel $h$ does not deviate if and only if $t_{H} / t_{L} \leq l_{2}$ which is equivalent to (2). Indeed, if $t_{H} / t_{L} \leq l_{2}$ then $\pi\left(p_{h}^{\prime}, p_{n}, p_{n}\right) \leq \pi\left(p_{n}, p_{n}, p_{n}\right)$ and so the deviation is unprofitable. This proves the "if" part. If $t_{H} / t_{L}>l_{2}$ then $t_{H} / t_{L}>l_{1}$ as well and so the deviation is profitable and $p_{h}^{\prime}>p_{n}+s t_{L}$, that is $h$ serves only business customers when it charges $p_{h}^{\prime}$. This proves the "only if" part. Therefore, $p_{n}$ is a symmetric equilibrium if and only if (2) holds.

Proof of Proposition 2. To prove the result, we proceed by contradiction. Assume that when $N \geq 4$ some high-type customers will choose opaque rooms in equilibrium.

Denote the list equilibrium price as $p_{H}$, and recall that the discounted price is equal to the marginal cost which is zero. The location of the business traveler who is indifferent between using opaque and nonopaque agencies is determined by the indifference condition:

$t_{H} x_{H}+p_{H}=\frac{1}{4} N s t_{H} \Rightarrow x_{H}\left(p_{H}\right)=\frac{N}{4} s-\frac{p_{H}}{t_{H}}$.

Similarly, an indifferent low-type customer is located at $x_{L}\left(p_{H}\right)=\frac{N}{4} s-\frac{p_{H}}{t_{L}}$.

Because some business travelers use the opaque service it has to be the case that $0<x_{H}<s / 2$. In what follows we will show that $x_{H}>s / 2$ when $N \geq 4$ which will be a contradiction to our initial assumption. 
The hotel's profit is equal to

$$
\pi\left(p_{H}\right)=\left\{\begin{array}{lr}
2 \frac{\gamma}{s} x_{H} p_{H} & \text { if } \left.x_{L}\left(p_{H}\right) \leq 0 \text { (i.e., } p_{H}>N s t_{L} / 4\right) \\
2 \frac{\gamma}{s} x_{H} p_{H}+2 \frac{1-\gamma}{s} x_{L} p_{H} & \text { if } \left.x_{L}\left(p_{H}\right)>0 \text { (i.e., } p_{H} \leq N s t_{L} / 4\right)
\end{array} .\right.
$$

The first line represents the case when all leisure travelers use the opaque agency, the second when some (but not all) of them use the opaque agency. Because $x_{L}<x_{H}<s / 2$ these are the only two cases.

If there is an equilibrium with $x_{H}<s / 2$ then the equilibrium price should maximize $\pi\left(p_{H}\right)$ within the range such that $0 \leq x_{H}\left(p_{H}\right)<s / 2$. There are three possible cases.

Case 1: $x_{L}\left(p_{H}\right)<0$ : all low-type customers prefer opaque product. Then the equilibrium price that maximizes hotel's profit is determined by

$\pi\left(p_{H}\right)=\max _{p_{H}} 2 \frac{\gamma}{S} x_{H} p_{H}$,

and from the FOC we have that

$p_{H}=\frac{N s t_{H}}{8}, \quad x_{H}\left(p_{H}\right)=\frac{N}{8} s$.

Clearly, $x_{H}\left(p_{H}\right)<s / 2$ only when $N<4$.

Case 2: $x_{L}\left(p_{H}\right)>0$. From (6) the hotel's profit is given by

$$
\begin{aligned}
\pi\left(p_{H}\right) & =2 \frac{\gamma}{s} x_{H} p_{H}+2 \frac{1-\gamma}{s} x_{L} p_{H} \\
& =2 \gamma\left(\frac{N}{4}-\frac{p_{H}}{s t_{H}}\right) p_{H}+2(1-\gamma)\left(\frac{N}{4}-\frac{p_{H}}{s t_{L}}\right) p_{H} .
\end{aligned}
$$

and it is maximized at

$$
p_{H}=\frac{1}{8} \frac{N s t_{L} t_{H}}{\gamma t_{L}+(1-\gamma) t_{H}} .
$$

Condition $x_{H}\left(p_{H}\right)<s / 2$ becomes:

$\frac{N}{4} s-\frac{1}{8} \frac{N s t_{L}}{\gamma t_{L}+(1-\gamma) t_{H}}<\frac{s}{2}$,

which is equivalent to

$2 t_{H}(1-\gamma)(N-2)<t_{L}(N-2 \gamma(N-2))$. 
When $N \geq 4, t_{H}$ is multiplied by a term that is greater than the term that multiplies $t_{L}$ and because $t_{H}>t_{L}$ (2) can hold only when $N<4$.

Case 3: $x_{L}\left(p_{H}\right)=0$. This case needs to be considered separately because it is possible that the profit function does not reach its maximum on intervals $p_{H}>N s t_{L} / 4$ and $p_{H}<N s t_{L} / 4$ in which case the FOC logic of Cases 1 and 2 is not applicable.

We will show that this case is impossible. Let $p_{0}$ be such price that $x_{L}\left(p_{0}\right)=0$. The profit function consists of two parabolas, and so in order for $p_{0}$ to bring the maximum it has to be the case that none of the two parabolas reach their maxima on the interval where they determine the profit. However, this is impossible. When $\pi\left(p_{H}\right)=2 \gamma s x_{H} p_{H}$, its maximum is reached at point $p_{H}=N s t_{H} / 8$, which is greater than $N s t_{L} / 4$ when $t_{H}>2 t_{L}$. If $t_{H} \leq 2 t_{L}$ then the second branch of the profit function reaches its maximum. Indeed, from (A4) we know that the maximum is reached at point

$p_{H}=\frac{1}{8} \frac{N s t_{L} t_{H}}{\gamma t_{L}+(1-\gamma) t_{H}}$.

To show that it is less than $N s t_{L} / 4$ is equivalent to showing that $t_{H} / 2<$ $\gamma t_{L}+(1-\gamma) t_{H}$, which is true because $t_{H} / 2 \leq t_{L}<\gamma t_{L}+(1-\gamma) t_{H}$.

Proof of Proposition 3.

(i) Assume that hotels $h-1$ and $h+1$ charge prices $p_{h-1}$ and $p_{h+1}$ respectively. Then hotel $h$ 's profit is

$\pi_{h}=\frac{\gamma}{s}\left(\frac{p_{h-1}+p_{h+1}-2 p_{h}}{2 t_{H}}+s\right) p_{h}$.

First order condition is

$0=\frac{\partial \pi_{h}}{\partial p_{h}}=\gamma\left(\frac{p_{h-1}+p_{h+1}-2 p_{h}}{2 t_{H}}+s\right)-\frac{\gamma}{t_{H}} p_{h}$.

Apply symmetry, we get

$p_{f}=s t_{H}$.

(ii) For $p_{f}=s t_{H}$ to be an equilibrium the incentive compatibility constraints should hold for both types and $p_{f}$ should maximize hotels' profit. Incentive compatibility for high type becomes

$s t_{H}+\frac{1}{2} s t_{H} \leq \frac{1}{4} N s t_{H}$, 
which holds if and only if $N \geq 6$. Incentive compatibility constraint for low type is

$$
\frac{1}{4} N s t_{L} \leq s t_{H}
$$

which holds if and only if $t_{H} / t_{L} \geq N / 4$. Later we will show that $\left(I C_{L}\right)$ will follow from the nondeviation conditions for the hotels.

The rest of the proof will determine the conditions under which hotels do not deviate. There are two possible deviations that should be considered. The first one is to participate in opaque sales but charge $p_{h} \neq s t_{H}$. The second one is to quit the opaque agency.

Claim 1. The first deviation is unprofitable if and only if

$\frac{t_{H}}{t_{L}} \geq \frac{N}{4}+\frac{1-\gamma}{2 \gamma}\left(\frac{N}{4}\right)^{2}$

Proof of Claim 1. We established in part (i) that $p_{f}$ is optimal for hotel $h$ if it serves only high-type customers. Thus the only potentially profitable price deviation for hotel $h$ is to decrease its list price so much that it would attract some leisure customers.

Hotel $h$ will attract leisure travelers if $p_{h}<N s t_{L} / 4$. Given that the two adjacent hotels charge price $p_{f}$, the profit function of hotel $h$ is given by

$$
\pi_{h}\left(p_{h}\right)=\left\{\begin{array}{lr}
\frac{\gamma}{s}\left(\frac{p_{f}-p_{h}}{t_{H}}+s\right) p_{h}+2 \frac{1-\gamma}{s} \frac{N s t_{L} / 4-p_{h}}{t_{L}} p_{h} & \text { if } p_{h}<\frac{1}{4} N s t_{L} \\
\frac{\gamma}{s}\left(\frac{p_{f}-p_{h}}{t_{H}}+s\right) p_{h} & \text { if } p_{h} \geq \frac{1}{4} N s t_{L}
\end{array}\right.
$$

This profit function is continuous and consists of two parabolas (see Figure 3). The first parabola represents hotel $h$ 's profit when some lowtype customers reserve rooms at hotel $h$, and the second one represents its payoff when it attracts no low-type customers.

As shown in part (i), the unrestricted maximum of the second parabola is reached at point $p_{f}=s t_{H}$ and from $\left(I C_{L}\right)$ we know that $p_{f}$ is greater than $\frac{1}{4} N s t_{L}$. Thus the profit function can achieve the global maximum at a price different from $p_{f}$ if and only if the first parabola reaches its maximum on the interval $\left[0,1 / 4 N s t_{L}\right]$ and its value at this maximum is greater than $\pi_{h}\left(p_{f}\right)$. 


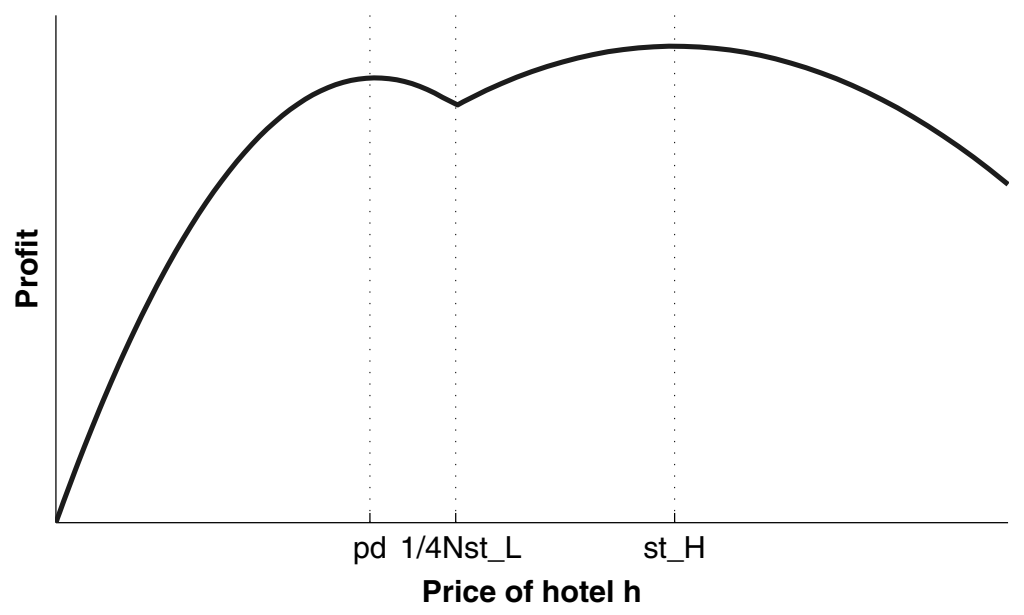

FIGURE 3. PROFIT FUNCTION OF HOTEL $h$

If we ignore for a moment the constraint $p \in\left[0,1 / 4 N s t_{L}\right]$, the maximum of the first parabola is reached at

$p_{d}=\frac{\gamma s t_{L} t_{H}+(1-\gamma) \frac{1}{4} N s t_{L} t_{H}}{t_{L} \gamma+2(1-\gamma) t_{H}}$.

When $p_{d}>\frac{1}{4} N s t_{L}$, the profit maximum is reached at point $p_{f}$, because the first parabola will be monotone on the range where it is defined. Thus the only local maximum $p_{f}$ will be also a global one. It is easy to see that condition $p_{d}>\frac{1}{4} N s t_{L}$ is equivalent to

$\left(1-\frac{1-\gamma}{\gamma} \frac{N}{4}\right) \frac{t_{H}}{t_{L}}>\frac{N}{4}$.

When (A7) is not satisfied the profit function has two local maxima. To find the global maximum we need to compare $\pi_{h}\left(p_{d}\right)$ and $\pi_{h}\left(p_{f}\right)$ $\pi_{h}\left(p_{d}\right) \leq \pi_{h}\left(p_{f}\right) \Leftrightarrow \frac{1}{s} \frac{\left(\gamma s t_{L}+(1-\gamma) \frac{1}{4} N s t_{L}\right)^{2}}{t_{L} \gamma+2(1-\gamma) t_{H}} \frac{t_{H}}{t_{L}} \leq \gamma s t_{H}$,

which reduces to $\frac{t_{H}}{t_{L}} \geq \frac{N}{4}+\frac{1-\gamma}{2 \gamma}\left(\frac{N}{4}\right)^{2}$.

A deviation where hotel $h$ changes the price without quitting the opaque agency is profitable if and only if (A7) and (A8) are violated. 
Notice that if $\frac{1-\gamma}{\gamma} \frac{N}{4} \geq 1$ then (A7) fails for sure, so hotel $h$ 's incentive to deviate is solely determined by (A8), which is precisely the condition stated in Claim 1. On the other hand, if $\frac{1-\gamma}{\gamma} \frac{N}{4}<1$, (A7) can be rewritten as

$\frac{t_{H}}{t_{L}} \geq \frac{N / 4}{1-\frac{1-\gamma}{\gamma} \frac{N}{4}}$.

It can be shown that

$\frac{N / 4}{1-\frac{1-\gamma}{\gamma} \frac{N}{4}} \geq \frac{N}{4}+\frac{1-\gamma}{2 \gamma}\left(\frac{N}{4}\right)^{2}$

and therefore, if condition (A8) is violated so is condition (A7). Again, whether hotel $h$ will deviate or not is completely determined by (A8). To summarize, if (A8) holds then hotel will not deviate because the deviation profit is less than the equilibrium profit. If (A8) does not hold then (A7) does not hold either and $h$ has a profitable deviation. This completes the proof of Claim 1.

ClaIm 2. Hotels will not withdraw from the opaque agency if and only if

$\frac{t_{H}}{t_{L}} \geq \frac{N}{N-1}\left(\frac{N}{4}+\frac{1-\gamma}{2 \gamma}\left(\frac{N}{4}\right)^{2}\right)$.

Proof of Claim 2. Assume that hotel $h$ wants to unilaterally quit the opaque agency. We calculate its profit from deviation, assuming that all other hotels do not change their behavior, whereas customers will respond optimally to new prices and the new opaque structure (this is similar to how, for example, Bertrand competition is analyzed).

In particular, all customers know that hotel $h$ will no longer provide opaque rooms, which changes their expected travel cost associated with the opaque agency. For the customer located at point $h$ the expected travel cost is equal to

$\frac{1}{N-1}\left(s+s+2 s+2 s+\cdots+\frac{N}{2}\right)=\frac{N}{4} \frac{N}{N-1} s=\frac{N^{2}}{4(N-1)} s$.

As we move away from point $h$, say to the left, the distance to $N / 2$ hotels on the left decreases although the distance to the remaining $(\mathrm{N}-$ 2) $/ 2$ hotels increases by the same amount. Thus the expected travel cost 
for the agent located at distance $x<N s / 2$ from hotel $h$ is

$$
\frac{N^{2}}{4(N-1)} s-\frac{N}{2} \frac{x}{N-1}+\frac{N-2}{2} \frac{x}{N-1}=\frac{N^{2}}{4(N-1)} s-\frac{x}{N-1} \text {. }
$$

The rest of the proof is similar to the proof of Claim 1. Given the result of part (i), hotel $h$ will not set a price such that only high-type customers are served. Thus, if the profitable deviation exists, hotel $h$ will serve customers of both types and will charge $p_{h}<p_{f}$.

The high-type consumer indifferent between hotels $h$ and $h-1$ is located at distance of $\left(s t_{H}-p\right) /\left(2 t_{H}\right)+s / 2$ from hotel $h$. Thus a total number of high-type agents who prefer hotel $h$ to either hotel $h-1$ or hotel $h+1$ is

$$
\frac{\gamma}{s}\left(\frac{s t_{H}-p}{t_{H}}+s\right) \text {. }
$$

In particular, notice that the largest group of high-type travelers that $h$ can potentially attract (if it charges price 0 ) is exactly the consumers located between hotels $h-1$ and $h+1$.

We observe that all high-type customers that prefer $h$ to adjacent hotels also prefer $h$ to the opaque agency and thus (A9) determines the number of high-type customers served by hotel $h$. Indeed, when $N \geq$ 6 and $p_{h}=p_{f}$ business travelers located at points $h-1$ and $h+1$ will prefer hotel $h$ to the opaque agency without hotel $h$. Thus, all customers between $h-1$ and $h+1$ will prefer hotel $h$ to the opaque agency without hotel $h$. This holds for any $p_{h}<p_{f}$ which is exactly the price range where profitable deviation is possible. Thus all high-type customers that prefer $h$ to $h-1$ and $h+1$ also prefer it to the opaque agency.

The leisure customer indifferent between hotel $h$ and the opaque agency is located at point $x$ such that

$\left(\frac{N^{2}}{4(N-1)} s-\frac{x}{N-1}\right) t_{L}=p+x t_{L}$

and so

$x=\frac{N}{4} s-\frac{N-1}{N} \frac{p}{t_{L}}$.

Thus a total number of low-type agents using the hotel $h$ is given by

$$
\frac{2(1-\gamma)}{s}\left(\frac{N s}{4}-\frac{N-1}{N} \frac{p}{t_{L}}\right)
$$


Hotel's profit is then

$\pi_{h}\left(p_{h}\right)=\frac{\gamma}{s}\left(\frac{s t_{H}-p_{h}}{t_{H}}+s\right) p_{h}+\frac{2(1-\gamma)}{s}\left(\frac{N s}{4}-\frac{N-1}{N} \frac{p_{h}}{t_{L}}\right) p_{h}$.

The maximum of the profit function is reached at point

$$
p_{\text {dev }}=\frac{\gamma+(1-\gamma) \frac{N}{4}}{\gamma t_{L}+2(1-\gamma) t_{H} \frac{N-1}{N}} s t_{H} t_{L},
$$

and the profit value at that point is equal to

$$
\pi_{d e v}=\frac{\left(\gamma+(1-\gamma) \frac{N}{4}\right)^{2}}{\gamma t_{L}+2(1-\gamma) \frac{N-1}{N} t_{H}} s t_{L} t_{H} .
$$

Similar to the logic used in proving Claim 1 we notice that the deviation is profitable if and only if $\pi_{d e v}>\gamma s t_{H}=\pi_{f}$ and $p_{d e v}<\frac{N^{2}}{4(N-1)} s t_{L}$. The last condition guarantees that when $p_{h}=p_{\text {dev }}$ leisure customers are served directly by hotel $h$. The expression on the right is the expected travel cost of a leisure customer located at point $h$ if he uses the opaque agency.

Condition $\left(\pi_{d e v}>\gamma s t_{H}=\pi_{f}\right)$ is violated when

$$
(1-\gamma)\left(\frac{N}{4}\right)^{2}+2 \gamma \frac{N}{4}-2 \gamma \frac{N-1}{N} \frac{t_{H}}{t_{L}}<0,
$$

which can be rewritten as

$$
\frac{t_{H}}{t_{L}} \geq \frac{N}{N-1}\left(\frac{N}{4}+\frac{1-\gamma}{2 \gamma}\left(\frac{N}{4}\right)^{2}\right) .
$$

Condition $p_{\text {dev }}<\frac{N^{2}}{4(N-1)} s t_{L}$ is violated when

$$
\frac{t_{H}}{t_{L}}\left(1-\frac{1-\gamma}{\gamma} \frac{N}{4}\right) \geq \frac{N}{4} \frac{N}{N-1}
$$

Notice that the only difference between (A8) and (A7) from Claim 1 and between (A10) and (A11) is that the right-hand side in the last two conditions is multiplied by $N /(N-1)$. Consequently, we can apply the reasoning of Claim 1 to show that hotel $h$ will not deviate if and only if (A10) holds. This completes the proof of Claim 2. 
Clearly, the condition in Claim 2 is more strict than the one in Claim 1. Furthermore, $\left(I C_{L}\right)$ follows from the condition provided by Claim 2. Combining our preceding arguments and the results of the two claims, we conclude that $p_{f}$ constitutes the equilibrium with full separation if and only if $N \geq 6$ and

$$
\frac{t_{H}}{t_{L}} \geq \frac{N}{N-1}\left(\frac{N}{4}+\frac{1-\gamma}{2 \gamma}\left(\frac{N}{4}\right)^{2}\right) \text {. }
$$

which completes the proof of Proposition 3.

Proof of Proposition 4. A slack $\left(I C_{H}^{\prime}\right)$ is equivalent to $p_{p s}<\frac{1}{4}(N-2) s t_{H}$. Using the expression (6) of $p_{p s}$, we can rewrite it as

$$
\frac{\gamma+\frac{1}{2}(1-\gamma) N}{\gamma t_{L}+4(1-\gamma) t_{H}} s t_{L} t_{H}<\frac{1}{4}(N-2) s t_{H} .
$$

By re-arranging terms we have

$$
\frac{t_{H}}{t_{L}}>\frac{\gamma+\frac{1}{2}(1-\gamma) N-\frac{1}{4}(N-2) \gamma}{(N-2)(1-\gamma)} \text {. }
$$

With some elementary calculations, we can show that the right hand side is less than 1 when $N \geq 6$. Because $t_{H} / t_{L}$ is higher than 1 , the above inequality is always valid. Therefore, $\left(I C_{H}^{\prime}\right)$ is not binding.

Proof of Proposition 5. From (6) we know that

$$
d^{\prime}=\frac{\frac{1}{4} N s t_{L}-p_{p s}}{t_{L}}=\frac{1}{4} N s-\frac{\gamma+\frac{1}{2}(1-\gamma) N}{\gamma t_{L}+4(1-\gamma) t_{H}} s t_{H} .
$$

Therefore, the partial separation requires

$$
0<\frac{1}{4} N s-\frac{\gamma+\frac{1}{2}(1-\gamma) N}{\gamma t_{L}+4(1-\gamma) t_{H}} s t_{H}<s / 2 .
$$

With some algebra, we can show:

$$
d^{\prime}<s / 2 \Leftrightarrow(N-2) \gamma t_{L}<2(\gamma(N-2)-(N-4)) t_{H}
$$

Notice that when $\gamma \leq \frac{N-4}{N-2}$, the right-hand side is less than zero and so the inequality cannot be satisfied. When $\gamma>\frac{N-4}{N-2}$ it has to be the 
case that

$\frac{1}{2} \frac{(N-2) \gamma}{\gamma(N-2)-(N-4)}<\frac{t_{H}}{t_{L}}$.

As for the condition $d^{\prime}>0$ we can show

$d^{\prime}>0 \Leftrightarrow\left(\gamma-\frac{1}{2}(1-\gamma) N\right) t_{H}<\frac{1}{4} N \gamma t_{L}$

When $\gamma \leq \frac{N}{N+2}$ then the left-hand side is nonpositive and so this inequality is automatically satisfied. If $\gamma$ is greater than $\frac{N}{N+2}$ then in order to have $d^{\prime}>0$, it must be the case that

$\frac{t_{H}}{t_{L}}<\frac{1 / 4 N \gamma}{\gamma-\frac{1}{2}(1-\gamma) N}$.

Collecting all these conditions together we have

$\frac{N-4}{N-2}<\gamma<\frac{N}{N+2}$ and $\frac{1}{2} \frac{(N-2) \gamma}{\gamma(N-2)-(N-4)}<\frac{t_{H}}{t_{L}}$

$\frac{N}{N+2}<\gamma<1$ and $\frac{1}{2} \frac{(N-2) \gamma}{\gamma(N-2)-(N-4)}<\frac{t_{H}}{t_{L}}<\frac{1}{4} \frac{N \gamma}{\gamma-\frac{1}{2}(1-\gamma) N}$.

This complete the proof.

Proof of Proposition 6.

(i) Denote ahotel's profit in the equilibrium with full separation as $\pi_{f}$ and the equilibrium without the opaque agency as $\pi_{n}$. We need to show that

$\pi_{f}=\gamma s t_{H}>\frac{s t_{H} t_{L}}{\gamma t_{L}+(1-\gamma) t_{H}}=\pi_{n}$.

It can be immediately seen that this is equivalent to

$\frac{t_{H}}{t_{L}}>\frac{1+\gamma}{\gamma}$.

(ii) Four conditions that determine the area where both equilibria exist and the full separation is more profitable are (2), $N \geq 6$, (3) and (7). We first show that (2) and (7) are not mutually exclusive. Condition (2) can 
be rewritten as

$$
\frac{t_{L}}{\gamma t_{L}+(1-\gamma) t_{H}}>\frac{1-\gamma / 2-\sqrt{1-\gamma}}{\gamma / 2},
$$

and (7) is equivalent to

$$
\frac{t_{L}}{\gamma t_{L}+(1-\gamma) t_{H}}<\gamma
$$

Thus in order for (2) and (7) to be consistent with each other it is necessary and sufficient that

$$
\frac{1-\gamma / 2-\sqrt{1-\gamma}}{\gamma / 2}<\gamma
$$

The numerator of the left-hand side is a strictly decreasing function and the denominator is strictly increasing. Thus the left-hand side is a strictly decreasing function. The right-hand side is a strictly increasing function and left-hand side $=$ right-hand side when $\gamma=1$. Thus the inequality holds for any $\gamma<1$.

The next step is to show that for any $N$ there are parameter values for which (2) and (3) are satisfied. Recall that condition (3) is

$$
\frac{t_{H}}{t_{L}} \geq \frac{N}{N-1}\left(\frac{N}{4}+\frac{1-\gamma}{2 \gamma}\left(\frac{N}{4}\right)^{2}\right) .
$$

As $\gamma \rightarrow 1$ this inequality becomes

$$
\frac{t_{H}}{t_{L}} \geq \frac{N^{2}}{4(N-1)} .
$$

At the same time (2) becomes $t_{H} / t_{L}<\infty$ as $\gamma \rightarrow 1$. When $\gamma=1$ these two conditions are consistent with each other. By continuity they are also mutually consistent for values of $\gamma$ that are close to 1 . Thus, for any $N \geq 6$ the set defined by (2), (3) and (7) is not empty.

(iii) To prove this part, we will proceed in two steps. We start by finding conditions under which $p_{p s}$ is higher than $p_{n}$, and then using Proposition 5 we will show that under these conditions the equilibrium with partial separation does not exist. 


\section{Step 1:}

$$
\begin{aligned}
p_{p s} & >p_{n} \\
& \Leftrightarrow \frac{\gamma+\frac{1}{2}(1-\gamma) N}{\gamma t_{L}+4(1-\gamma) t_{H}} s t_{L} t_{H}>\frac{s t_{L} t_{H}}{\gamma t_{L}+(1-\gamma) t_{H}} \\
& \Leftrightarrow \frac{1}{2} \gamma N t_{L}+\frac{1}{2}(1-\gamma) N t_{H}+\gamma t_{H}>\gamma t_{L}+4 t_{H} \\
& \Leftrightarrow t_{L} \gamma(N-2)>t_{H}(\gamma(N-2)-(N-8)) .
\end{aligned}
$$

The right-hand side is positive whenever $\gamma>\frac{N-8}{N-2}$. For the partial equilibrium to exist it has to be the case that $\gamma>\frac{N-4}{N-2}$ so the right-hand side is positive whenever the partial separation equilibrium exists. Thus,

$p_{p s}>p_{n} \Leftrightarrow \frac{t_{H}}{t_{L}}<\frac{\gamma(N-2)}{\gamma(N-2)-(N-8)}$.

Step 2: As it is shown in Proposition 5, for partial equilibrium to exist it has to be the case that

$$
\frac{t_{H}}{t_{L}}>\frac{1}{2} \frac{\gamma(N-2)}{\gamma(N-2)-(N-4)} \text {. }
$$

However, as we are going to show, these two inequalities (A12) and (A13) are mutually exclusive. Indeed,

$$
\begin{aligned}
\frac{1}{2} \frac{\gamma(N-2)}{\gamma(N-2)-(N-4)} & >\frac{\gamma(N-2)}{\gamma(N-2)-(N-8)} \\
& \Leftrightarrow-\frac{1}{2}(N-8)>\frac{\gamma}{2}(N-2)-(N-4) \\
& \Leftrightarrow N>\gamma(N-2),
\end{aligned}
$$

which always holds because $\gamma \leq 1$.

Thus, whenever the equilibrium with partial separation exists its price $p_{p s}$ is less than the price without the opaque agency $p_{n}$. Moreover, in the former case some customers are charged marginal cost whereas in the latter case all customers pay price $p_{n}$. Thus hotel's profit is higher in the equilibrium without the opaque agency.

\section{REFERENCES}

Armstrong, M., 2006, "Recent Developments in the Economics of Price Discrimination," inR. Blundell, W. Newey, and T. Persson, eds., Advances in Economics and Econometrics: Theory and Applications, pp. 97-141. Vol. 2, Cambridge, UK: Cambridge University Press. 
Cooper, T.E., 1986, "Most-Favored-Customer Pricing and Tacit Collusion," RAND Journal of Economics, 17(3), 377-388.

Deneckere, R.J. and R.P. McAfee, 1996, "Damaged Goods," Journal of Economics and Management Strategy, 5, 149-174.

Ellison, G., 2005, "A Model of Add-on Pricing," Quarterly Journal of Economics, 120(2), 585-637.

Fay, S., 2004, "Partial-Repeat-Bidding in the Name-Your-Own-Price Channel," Marketing Science, 23, 407-418.

, 2008, "Selling an Opaque Product through an Intermediary: The Case of Disguising One's Product," Journal of Retailing, 84(1), 59-75.

Granados, N., A. Gupta, and R.J. Kauffman, 2005, “Transparency Strategy in Internetbased Selling," in K. Tomak, ed., Advances in the Economics of Information Systems, Harrisburg, PA: Idea Group Publishing, 80-112.

Hann, I. and C. Terwiesch, 2003, "Measuring the Frictional Costs of Online Transactions: The Case of a Name-Your-Own-Price Channel," Management Science, 49, 1565-1581.

Holt, C.A. and D.T. Scheffman, 1987, "Facilitating Practices: The Effects of Advance Notice and Best-Price Policies," RAND Journal of Economics, 18, 187-197.

Hotelling, H., 1929, "Stability in Competition," Economic Journal 39, 41-57.

Krishna, K., 1989, "Trade Restrictions as Facilitating Practices," Journal of International Economics, 26, 251-270.

Salop, S., 1979, "Monopolistic Competition with Outside Goods," The Bell Journal of Economics, 10, 141-156.

Scott Morton, F., 1997, "The Interaction between a Most-Favored-Customer Clause and Price Dispersion: An Empirical Examination of the Medicaid Rebate Rules of 1990," Journal of Economics and Management Strategy, 6, 151-174.

— 1997, "The Strategic Response by Pharmaceutical Firms to the Medicaid MostFavored-Customer Rules," RAND Journal of Economics, 28, 269-290.

Spann, M., B. Skiera, and B. Schafers, 2004, "Measuring Individual Frictional Costs and Willingness-to-Pay via Name-Your-Own-Price Mechanisms," Journal of Interactive Marketing, 18(4), 22-36.

Stole, L., 2007, "Price Discrimination and Competition," in M. Armstrong, and R. Porter, eds., Handbook of Industrial Organization, pp. 2221-2300. Vol. III, Amsterdam: NorthHolland.

Terwiesch, C., S. Savin, and I. Hann, 2005, “Online Haggling at a Name-Your-Own-Price Retailer: Theory and Application," Management Science, 51, 339-351.

Wang T., E. Gal-Or, and R. Chatterjee, 2006, "When Should a Service Provider Employ a 'Name Your Own Price' Channel?" Working Paper, University of Pittsburgh, Pittsburgh, PA. 